\title{
Processus et composition
}

\section{Thierry Smolderen}

\section{(2) OpenEdition \\ Journals}

Édition électronique

URL : http://journals.openedition.org/genesis/1690

DOI : 10.4000/genesis. 1690

ISSN : 2268-1590

\section{Éditeur :}

Presses universitaires de Paris Sorbonne (PUPS), Société internationale de génétique artistique littéraire et scientifique (SIGALES)

\section{Édition imprimée}

Date de publication : 12 décembre 2016

Pagination : 65-85

ISBN : 9791023105490

ISSN : 1167-5101

\section{Référence électronique}

Thierry Smolderen, «Processus et composition », Genesis [En ligne], 43 | 2016, mis en ligne le 28 octobre 2017, consulté le 04 mai 2019. URL : http://journals.openedition.org/genesis/1690 ; DOI : 10.4000/genesis. 1690

Ce document a été généré automatiquement le 4 mai 2019.

Tous droits réservés 


\title{
Processus et composition
}

\author{
Thierry Smolderen
}

\section{Définir un sujet et commencer à construire}

1 Avant l'écriture elle-même (sur ordinateur), je passe par une très longue phase d'accumulation d'éléments qui tournent autour d'un thème, d'une image forte, d'un ressort romanesque, d'une période stylistique, d'une œuvre de référence ou d'un genre (et parfois, le tout en même temps). Quelque chose (parfois de l'ordre du détail) a suscité en moi une émotion initiale, un moment d'euphorie qui tient un peu du «coup de foudre ». L'envie (assez irrépressible) de partager ce sentiment et de le prolonger par le biais d'une bande dessinée définit le périmètre initial de chaque projet.

2 Pour donner un exemple récent (L'Été Diabolik, avec Alexandre Clérisse ${ }^{1}$ ), l'idée de cet album est née du désir de travailler avec Alexandre sur une période graphique différente de celle que nous avions explorée dans notre livre précédent (le «style atome» des années cinquante). Depuis quelque temps, je passais en revue des films et des BD de la fin des années soixante, dont l'esthétique pop faisait naître en moi bien des envies, et le glissement vers l'univers visuel d'Alexandre Clérisse s'est fait tout naturellement.

Un jour, une image toute simple m'est venue en tête : un homme - un industriel, peut-être jette un coup d'œeil dans son rétroviseur, et aperçoit, au volant de la voiture qui le suit, la silhouette de Diabolik, le criminel masqué des fumetti italiens des années soixante. Cette vision fantastique disparaît aussitôt, mais quelques jours plus tard, l'expérience se reproduit... Et il ne comprend pas pourquoi.

4 Le prétexte était mince, mais une trouvaille du magnifique film pop de Mario Bava, Danger : Diabolik (1968), visionné dans la foulée, l'avait considérablement renforcée : on y voit le personnage de Diabolik conduire sa Jaguar Type-E à visage découvert. Un rayon de soleil, frappant son rétroviseur, illumine le visage du conducteur, et l'espace d'une seconde ce rectangle de lumière lui projette un masque virtuel autour des yeux.

5 Tout le scénario s'est développé autour de ce jeu de réfraction: l'idée d'un masque sautant d'un personnage à l'autre, comme s'il cherchait un acteur suffisamment « diabolique » pour l'incarner. 
6 J'utilise couramment le cinéma pour m'imprégner des genres, des périodes et des styles dans lesquels mes histoires s'enracinent. Je passe en revue des dizaines de films, à la recherche d'idées visuelles qui pourraient me dire quelque chose de pénétrant sur le regard et l'imaginaire de l'époque. Un décor, un cadrage, une lumière, une pose, un geste, un costume peuvent provoquer l'étincelle, éclairer d'un jour nouveau une situation dramatique dont je n'avais encore qu'une idée assez vague et abstraite - ou pas d'idée du tout.

7 La BD, mieux encore que le cinéma, stylise et schématise. Sous un léger vernis, elle superpose, comme autant de "calques", des plans d'expression sémiotiquement très ciblés qui sont différentes «façons de voir » le monde à travers des lentilles spécialisées. La forme de la bulle, par exemple, peut marquer des différences subtiles entre des sources d'énonciation très variées (message passant à la radio, cri collectif, onomatopée, parole d'être humain ou de robot, etc.) ; dans une même histoire, le dessin peut adopter des lentilles stylistiques très hétérogènes et en jouer, comme le roman le fait avec différentes «façons de parler».

8 Je suis sensible à cette dimension prismatique de l'image de bande dessinée. Elle m'est naturelle, comme elle l'était à des gens comme Pierre Sterckx, Henri Van Lier et d'autres, que j'ai eu la chance de fréquenter pendant mes études artistiques à la Cambre dans les années soixante-dix. J'ai appris auprès d'eux que la bande dessinée était un très bon révélateur, analytique et affectif, des composants de l'image du $\mathrm{xx}^{\mathrm{e}}$ siècle. Comme le cinéma burlesque, elle a eu le privilège d'entrer très tôt dans la vie de son public, et elle a été source de mille petites épiphanies visuelles pour les générations nées au milieu du siècle.

9 Historiquement, la dimension polygraphique de l'image de bande dessinée vient du dessin humoristique, et elle s'est manifestement communiquée au cinéma (sans doute vers le milieu de l'époque du muet).

Dominique Bertail m'a beaucoup aidé à aborder l'image cinématographique, notamment en me faisant prendre conscience de la dimension graphique du travail sur la lumière. Nous avons mis ce «calque " à profit pour informer les thématiques visuelles de notre thriller Ghost Money et désormais je me livre à ce genre de déconstruction (hautement jubilatoire) chaque fois qu'un scénario m'entraîne vers une période stylistique donnée.

11 Beaucoup de réalisateurs (et de directeurs photos) construisent l'image de cette façon, par superposition de couches sémiotiques. Il suffit, par exemple, de voir comment David Lynch, pour inventer le monde de Twin Peaks, a déconstruit le film Peyton Place en captant différents « calques » du prototype (la gestuelle des acteurs, certains personnages dont il a gardé les traits distinctifs, certaines situations, quelques images clés, etc.).

12 Le travail préparatoire que j'effectue sur le graphisme, le cinéma, le design, la publicité, l'illustration, déborde donc largement la fonction documentaire.

13 Chaque projet de scénario donne lieu à une sorte de dossier mental dans lequel j'entrepose, au fil du temps, des idées dramatiques originales qui me stimulent. Elles peuvent sembler très disparates, pourtant elles sont "apparentées », un peu comme les reliques d'une même famille se sédimentent dans un grenier. Dans certaines histoires, les souvenirs et les fantasmes intimes peuvent d'ailleurs jouer un rôle important.

14 Je promène toujours ce "dossier " avec moi, et dès que je peux y déposer un nouvel ingrédient- fruit d'une rencontre, d'une lecture, de la vision d'un film, d'une expérience vécue, d'une réminiscence, d'une association d'idée (éventuellement théorique) - je 
l'engrange précieusement, mais sans prendre la moindre note. Je confie tout cela à un tiroir réservé de ma mémoire - c'est la dimension organique du processus, si l'on veut.

Cette première phase concerne vraiment le matériau de base de l'histoire. Elle s'enracine dans mes intérêts profonds, mes intuitions enfantines, certaines préoccupations fondamentales qui se cristallisent là, sous forme de personnages, de séquences, de décors, de dispositifs.

Toute la difficulté vient du fait que je ne veux ranger dans ce dossier virtuel que des fragments prégnants, des bribes d'idées, qui, prises isolément, possèdent déjà, à mes yeux, de fortes résonances personnelles et/ou un riche potentiel dramatique et romanesque.

17 Cette phase peut s'étaler sur une très longue durée (deux ou trois ans pour le scénario de L'Été Diabolik, plus de vingt ans pour Souvenirs de l'empire de l'atome ou McCay) - et durant tout ce temps je me garde bien de faire des choix qui engageraient déjà la narration sur un rail. Si j'ai une situation forte, un personnage intéressant, un bon début d'histoire ou un rebondissement possible, j'évite scrupuleusement d'en fixer les causes ou les conséquences dramatiques, avant d'avoir une vision d'ensemble.

J'attends que mon "grenier" soit suffisamment saturé d'idées prometteuses pour vraiment commencer à monter ma petite pièce de théâtre, comme le feraient des enfants qui se déguisent et improvisent à partir d'accessoires accumulés un peu au hasard (mais qui reflètent l'histoire d'une "même famille " et donc, une même famille d'histoires). Cette sédimentation se termine quand j'entrevois subitement la forme d'un arc narratif que je juge « assez bon " pour commencer à raconter.

Dans L'Été Diabolik, par exemple, l'idée qui a joué ce rôle catalytique a été de centrer le scénario sur les doutes qu'un adolescent commence à éprouver vis-à-vis de la personnalité de son père. Tout de suite, elle s'est imposée à moi comme la meilleure épine dorsale possible pour les éléments que j'avais déjà rassemblés : l'été 1967 ; un industriel entouré de menaces ; le masque de Diabolik sautant d'un personnage à l'autre ; l'esprit de la bande dessinée et du film d'espionnage des années pop. Condition sine qua non, le thème offrait aussi de vraies possibilités de dénouement.

Quand ce genre d'ampoule s'allume au-dessus de ma tête, je boucle définitivement le périmètre : je considère que j'ai mon matériau de base. Il ne s'agit plus d'ajouter quoi que ce soit de fondamentalement nouveau (un autre enjeu majeur) qui risquerait de compliquer les choses. Je n'introduirai plus que des idées qui ont vocation à condenser et simplifier ces données originales, et à les articuler entre elles.

\section{Le tressage dramatique}

21 Vient alors une phase assez épuisante - et sans doute la plus technique du processus en cours - qui consiste à combiner ces éléments de toutes les manières imaginables en en explorant toutes les facettes.

Ma base de données est franchement hétéroclite: j'entrevois un ou deux thèmes dramatiques majeurs, des ébauches de personnages, des amorces de motivation, d'enjeux romanesques et de rebondissements, des lieux et des ambiances exploitables. Mais tout cela cohabite «en suspension ", sans véritables liens logiques, et il va falloir faire évoluer le système par petites touches, en testant mille et une hypothèses. 
23 L'arc narratif qui m'a fait passer à cette étape n'est encore qu'une première approximation. Je m'y réfère de manière souple et ouverte, sachant que le plan de vol peut changer radicalement en cours de route. En fait, la seule chose dont je me préoccupe dès le départ, est de faire évoluer ma conception du climax de l'histoire. Au bout de cet arc, j'ai entrevu un dénouement «suffisamment bon » qui m'a encouragé à boucler la phase précédente, mais ce dénouement est loin d'être fixé. Une idée tout à fait différente peut très bien survenir tardivement et changer le sens profond du récit tout entier (un autre point de vue, un autre personnage central, etc.). Mon espace de travail est donc vectorisé. À l'horizon, il y a toujours cette petite lumière vacillante - un dénouement possible - qui indique une direction.

24 À tous les niveaux, et à toutes les étapes du travail, je cherche à préserver la plus grande marge de liberté possible. J'assemble des bribes, je saute d'une petite poche d'ordre à l'autre en reconfigurant sans cesse la géographie de l'ensemble. La métaphore du puzzle s'applique assez bien à mon approche par essais et erreurs - à cette différence près que l'image complète du puzzle ne préexiste pas à l'opération.

$\mathrm{Au}$ bout du compte, pourtant, il faudra bien trouver une « solution linéaire » à ce cabinet de curiosités - l'équation narrative la plus logique, la plus économique et la plus efficace $\mathrm{du}$ point de vue dramatique, qui permettra de faire entrer une bonne partie de son contenu dans les rails d'un récit séquentiel.

Du point de vue génétique, la fonction de ce long processus d'incubation est d'échapper à la fatalité des enchaînements de causes et d'effets prévisibles, de ces scripts tout faits, qui se présentent spontanément à l'esprit quand on développe une situation romanesque de manière linéaire (et en particulier, dans le périmètre d'un genre bien établi où tous les plis, toutes les séquences « classiques ", sont déjà bien définis). Construire une histoire en essayant de tirer logiquement les conséquences d'une situation de départ (aussi prometteuse soit-elle) est comparable à ce qui se passe quand nous parlons sans être totalement impliqué dans ce que nous disons : les mots s'associent machinalement, dans des formules qui canalisent l'expression de notre pensée. À mesure que le syntagme se complète, le spectre de choix disponibles (sur le plan syntactique et sémantique) rétrécit drastiquement. Si l'on ne se défend pas contre cette logique aveugle, on conclut par une platitude. Ou la phrase s'avère sans issue et s'embourbe lamentablement. De même pour les histoires...

27 Pour contrer cette tendance, j'exploite le privilège de la vue simultanée, de la vue en surplomb du matériau sur lequel je travaille : je laisse délibérément des trous béants, des fils dénoués dans le tissu de l'histoire en chantier.

Des segments majeurs de l'intrigue m'échappent encore, et, dans les quelques scènes qui commencent à se préciser sur le plan dramatique, il reste mille petites choses à régler : Pourquoi décide-t-elle de sortir de chez elle à ce moment-là ? Dans quelles circonstances les deux héroïnes vont-elles se rencontrer? Quelle arme utilise-t-il pour le tuer?...

Les questions de ce type sont innombrables dans un scénario, et je combats la tentation d'y répondre machinalement par des solutions qui n'ont pour elles que d'être vraisemblables et utiles. Car, très souvent, en explorant des solutions non standard (jusqu'au moindre détail), on tombe sur des idées dont les prolongements se révèlent très fertiles, voir déterminants pour l'ensemble de l'intrigue.

30 Par ailleurs, ce réseau de lacunes, de fils flottant librement, peut être tourné à l'avantage du scénariste. Quand on surplombe le scénario, tous ces points d'interrogations 
d'importance variables offrent un nombre incalculable de configurations possibles. Après tout, ils coexistent dans le même monde, et il ne tient qu'à moi de profiter de cette vaste terra incognita pour inventer de nouvelles connexions.

31 Comme dans l'assemblage d'un puzzle, l'espace négatif de l'histoire (le "pays » des questions en suspens) joue donc un rôle aussi important que les pièces elles-mêmes. Car il ne s'agit jamais de déterminer une solution à un problème unique (sous forme d'une justification linéaire, d'une explication), mais d'en résoudre plusieurs à la fois - de faire des strikes qui en éteignent toute une constellation d'un seul coup.

De là l'intérêt de préserver des options et questions en suspens aussi longtemps que possible. En combinant ces lacunes, en testant des scripts alternatifs ou en cherchant à intégrer des «objets trouvés» qui me semblent prometteurs, il m'arrive d'entrevoir subitement une corrélation qui m'avait échappé auparavant: la possibilité d'utiliser une clé commune pour débloquer tout un groupe de problèmes.

Dans L'Été Diabolik, par exemple, le père disparaitt mystérieusement suite à une série de péripéties inexplicables. On comprendra tout à la fin que la personnalité « de façade » du père dissimule une réalité très sombre, mais son masque est si bien ajusté que le fils ne s'en aperçoit pas consciemment. Du point de vue scénaristique, la difficulté était de manifester ce double-fond dans le comportement du père. Comment créer autour de lui une vague aura de malaise tout en le faisant agir de manière crédible dans la vie quotidienne?

Posé de cette manière, le problème parait assez bien défini pour qu'on puisse l'aborder méthodiquement, suivant les plis bien établis du genre policier ou d'espionnage. Par exemple, en partant du secret du père pour inventer quelques indices révélateurs de sa double vie. Mais c'est typiquement ce que j'évite de faire.

Je préfère laisser « flotter » mon attention autour de la question, en gardant à l'esprit les nombreuses autres lacunes qui me restent à combler : dans ce cas précis, l'apparence physique du père et son comportement, les liaisons logiques entre certaines scènes « à faire ", les incidents inattendus à partir desquels je cherchais à décrire le quotidien du jeune héros en vacances, sa relation avec d'autres personnages, etc. (la liste est loin d'être exhaustive).

La solution m'est venue dans une librairie d'Angoulême, où j'ai trouvé, en cherchant tout autre chose, une collection de luxueuses revues de mode datant du début des années soixante, Numéro Un, le magazine de l'élégance masculine française.

En les feuilletant, j'ai éprouvé ce petit choc émotionnel typique de l'objet trouvé. Ces revues nous offraient une documentation visuelle très riche dans un domaine difficile d'accès (l'élégance masculine française des années soixante), des photographies couleur de très bonne qualité montrant des costumes, des chaussettes, des cravates, des chapeaux, des boutons de manchettes (quelques échantillons de tissu étaient même insérés entre les pages). J'insiste là-dessus car l'aspect parfaitement inopiné de cette trouvaille (qui m'offrait des éléments que j'aurais eu très peu de chances de trouver si je les avais cherchés), ne pouvait que chatouiller ma fibre opportuniste.

Immédiatement, j'ai pensé à l'apparence physique du père (pour laquelle Alexandre Clérisse et moi n'avions encore rien défini) ; presque dans le même instant, j'ai pensé au malaise diffus qu'il était censé inspirer à son fils (et au lecteur). Je me suis aussi souvenu d'une scène de mon adolescence, une remarque faite en passant par un copain qui m'avait dit un jour qu'il trouvait mon père " impressionnant », ce qui m'avait laissé très perplexe. 

dramatiques et romanesques, je vais donc chercher un fil conducteur capable de me sortir $\mathrm{du}$ labyrinthe, et de relier logiquement tous ces segments épars. Pour y tester les différentes liaisons causales possibles, je devrai résolument faire mienne la logique de l'action progressive, entrer dans un espace dynamique, pleinement vectorisé. autonome sur le plan dramatique, avec son point d'entrée et son point de sortie. Quand je parle de tirer un fil qui exploite au mieux le potentiel multimodal de mon fatras d'idées, je pense essentiellement à cette tâche, qui consiste à décrire l'arc narratif uniquement en termes de séquences de longueurs variables (une à cinq pages, en général) et qui auront aussi, c'est important, une unité visuelle très accentuée (lumière, décor, couleur). nombreuses. La séquence est conçue comme un mini-récit à part entière : un point d'entrée (le plus dynamique possible), un développement, une conclusion (qui fonctionne comme une ponctuation, et projette le lecteur vers le point d'entrée de la séquence suivante).

pleinement le caractère modulaire de cette microstructure très simple, très vectorisée. Contrairement à Hergé et à beaucoup d'autres scénaristes du milieu du $\mathrm{xx}^{\mathrm{e}}$ siècle, je ne cherche pas à masquer les ellipses pour suggérer un semblant de continuité temporelle ou spatiale.

La pâte n'est pas lisse. Les coutures de l'assemblage resteront visibles, parfois même, je les soulignerai délibérément. Le lecteur ne pourra pas douter qu'il s'agit d'un patchwork. Ce que l'on perd en réalisme (au sens classique d'une représentation mimétique de la continuité du temps et de l'espace), on peut le regagner en termes d'empowerment ludique : le montage des séquences laisse transparaitre une forme d'énergie, un rythme 
subjectif qui n'appartient qu'au conteur de la fable - une " prise en main ", tantôt douce, tantôt vigoureuse, tantôt ironique, de l'espace-temps élastique de ce petit monde.

À l'intérieur de chaque séquence, cependant, je m'efforce de préserver l'illusion de la continuité en intégrant les différents enjeux de l'épisode (qui peuvent être d'ordre très divers et impliquer des registres d'expression très hétérogènes) dans une suite d'incidents qui paraîtra la plus linéaire, la plus naturelle et fluide possible. J'orchestre l'information en fonction des points forts de la scène, et pour servir au mieux sa conclusion.

48 À ce stade, cependant, ce n'est encore qu'un brouillon : un aménagement « suffisamment bon » du contenu de la séquence, qu'il faudra soumettre au test de l'écriture et du découpage pour voir, en particulier, s'il loge dans l'espace imparti. Les séquences doivent être courtes. Très souvent, je me verrai obligé de sacrifier des idées ou des développements secondaires pour qu'elles ne deviennent pas d'interminables « tunnels». Ce qui rend l'unité de la séquence si importante à mes yeux, c'est qu'elle incarne le "présent de la lecture »: l'expérience intense d'un ici et maintenant, comparable à ce que nous vivons quand tous nos sens, toutes nos facultés cognitives, sont mis en alerte par une situation hors normes.

50 La structure synoptique du récit, pour sa part, relève d'un tout autre genre d'organisation. Son unité n'apparaît (forcément) qu'après une première lecture complète : elle est toujours rétrospective et synthétique - et plutôt normative.

51 L'importance accordée au "présent de la lecture » joue un rôle déterminant dans la manière de construire un scénario. Jacques Martin, qui fut longtemps le collaborateur d'Hergé, s'étonnait souvent de l'énergie qu'il mettait à rechercher des gags pour nourrir chaque séquence, comme s'il se préoccupait plus de ces détails ponctuels que de la structure générale de l'histoire. En fait, l'étude de rushs de Chaplin montre que le cinéma muet (qui avait bercé l'enfance du père de Tintin) procédait souvent de manière similaire. Dans les films de Chaplin, l'arc général de l'histoire ne se décidait souvent qu'à la fin d'un long processus d'improvisation tous azimuts.

Cette façon de faire creuse un fossé entre l'expérience même de la lecture et sa paraphrase "après coup ». Les deux niveaux ne sont pas du tout vécus de la même manière par le lecteur. Si vous lui demandez de résumer Tintin au Tibet, par exemple, il vous dira invariablement que l'histoire raconte comment Tintin, convaincu de la survie de son ami Tchang, est parti au Tibet pour essayer de le retrouver, ce qu'il finit par faire en remontant jusqu'à l'antre du Yéti où son ami était retenu prisonnier.

En réalité, le vrai moteur de l'histoire, qui nourrit pratiquement chaque séquence de l'album, est la résistance qu'oppose Haddock à ce projet irrationnel de sauvetage. Tous les incidents, toutes les actions de l'aventure se rapportent à ce conflit amical, constamment réactivé et sous tension.

Si l'on peut relire Tintin indéfiniment, c'est parce que l'expérience immersive de la lecture - qui se joue réellement à l'échelle de chaque séquence - est si différente de ce qu'on appelle le pitch de l'histoire. Celui-ci est facile à exposer verbalement, tandis que celle-là ne peut être vécue que sur le mode de l'image, de l'action - et bien sûr, du gag -, le tout fonctionnant comme une sorte de réaction en chaîne courant de la première à la dernière image de l'album.

Mon «esthétique » de l'intrigue reprend cette formule mais en la compliquant. Il faut dire que les genres dans lesquels je m'exprime le mieux (le thriller, la science-fiction, etc.) 
se prêtent bien à ce type de complication - mais il y a un prix à payer : au grand désarroi de mes éditeurs, mes histoires sont pratiquement impossibles à résumer, ou, comme ils disent, à pitcher.

\section{Réaction en chaîne}

La conception de la conduite séquentielle de l'histoire s'accompagne de la production de nombreux diagrammes - plusieurs dizaines pour certains albums, à mes débuts - qui représentent le fil de l'action.

Une ligne figure l'ensemble de l'album: c'est mon plan. Je le segmente en séquences qu'un court descriptif suffit à préciser. J'attribue (au jugé) un nombre de pages à chaque séquence.

Chaque nouveau plan essaye d'intégrer des liaisons inédites entre les scènes. Je déplace ou supprime une séquence, j'en invente une autre, etc.. Immédiatement des problèmes imprévus apparaissent et je bricole une version alternative pour essayer d'y remédier.

Quand un plan me paraît suffisamment " robuste » à cette échelle de travail, je le mets réellement à l'épreuve dans le détail, en me projetant mentalement à l'intérieur de la galerie séquentielle qu'il décrit. Ma tâche est, à présent, de suivre scrupuleusement le flux de l'information et en repérer les points faibles. Telle explication nécessaire pour justifier une scène sera indigeste, l'exposition de telle situation, trop frontale ou sans éclat; les motivations de tel personnage sont franchement peu crédibles, voire inexistantes, etc.

Dans la conception même des séquences, la gestion des "explications " joue un rôle primordial. Par définition, une histoire intriquée - sauf si elle est tissée de clichés demande beaucoup d'éclaircissements et de justifications. Pour pouvoir maintenir le lecteur sous tension par des rebondissements, des surprises, de nouveaux mystères, l'intrigue doit progresser continuellement, mais elle doit le faire de manière implicite, c'est-à-dire, avec le minimum d'exposition discursive. Même si le résultat final donne l'impression d'une certaine complexité, les relations de cause à effet (prises isolément) doivent « s'expliquer d'elles-mêmes », ou du moins, en donner l'impression.

61 Cela ne signifie pas qu'il faut éviter les dialogues, bien au contraire, mais ceux-ci doivent fonctionner exactement comme une action. (C'est en cela que la tension amicale entre Haddock et Tintin joue véritablement un rôle moteur dans Tintin au Tibet.)

62 La tension dramatique ne peut pas se relâcher subitement pour exposer les choix opérés par les personnages. Idéalement, chaque échange verbal prend la forme d'une réaction en chaîne tendue vers un but: les mots sont réifiés, les répliques exercent des forces exactement comme des armes, des leviers, des objets ; chaque dialogue peut alors devenir une petite aventure avec ses surprises et ses rebondissements.

63 À mes heures perdues, il m'arrive souvent d'imaginer le cours que pourrait prendre tel ou tel dialogue et d'en noter quelques phrases au revers d'une enveloppe quand j'entrevois soudain le moyen d'insérer naturellement une série d'informations indispensables dans une réaction en chaîne de ce genre. Les passages les plus «informatifs » (donc, les plus difficiles) du dialogue de l'album sont ainsi réglés bien avant l'écriture du scénario. Ils font pièce avec l'action.

64 L'autre problème essentiel porte sur la nature du «crochet » que l'on va lancer à la fin d'une séquence pour sauter vers le début de la séquence suivante. 
65 Là encore, le risque est grand de se reposer sur des automatismes génériques, en introduisant paresseusement des scènes de transitions attendues, ou purement explicatives. Personnellement, j'aime profiter du saut dans le vide que représente le passage d'une séquence à l'autre pour ruser avec le lecteur - par exemple, en éliminant toute une série d'actions intermédiaires prévisibles, pour l'emmener plus directement qu'il ne prévoyait vers les conséquences de ce qui vient d'être établi - ou pour l'emmener tout à fait ailleurs. Tout dépend aussi du degré d'ironie ou d'intervention auctoriale que l'on veut glisser dans l'interstice (car les ellipses sont, typiquement, l'endroit où l'on peut, sans frais, laisser entrevoir fugitivement la main ou le sourire du marionnettiste).

Il m'arrive, cependant, de rencontrer de véritables impasses à ce stade : dans certains cas, malgré tous mes efforts, je ne trouve pas le moyen de passer élégamment d'un point à un autre. C'est rarement une lacune de logique qui me pose les problèmes les plus insurmontables. En général, la scène $\mathrm{B}$ répond parfaitement à la scène $\mathrm{A}$ - mais justement, le lien de cause à effet entre les deux me paraît trop frontal, trop délibéré : c'est un câble. Si la raison d'être des deux scènes est étriquée, univoque, si elle se résume à un seul enchaînement de faits (fût-il surprenant et crucial pour l'intrigue), cela ne me laisse aucune marge de manœuvre. Car (contrairement à ce que croyait Jacques Martin!), ce n'est pas ce qui se passe qui est important. Sur le plan dramatique, la seule chose qui compte, c'est comment ça se passe.

D'expérience, je sais que les blocages de ce genre se produisent parce que l'espace négatif du segment en question est verrouillé : je n'ai pas eu à résoudre assez de petits problèmes entre ces deux scènes pour y répondre par un tressage de solutions obliques à de multiples niveaux.

La scène $\mathrm{A}$ pose une question frontale, et la scène $\mathrm{B}$ y répond, c'est tout. Le bloc se suffit à lui-même, et c'est ce qui le rend stérile. Il n'offre aucune surface de résonance avec d'autres aspects de l'histoire et donc aucun moyen de jouer et de ruser avec la dramatisation.

Pour sortir de ce type d'impasse, j'ai recours à un procédé graphique ad hoc. Je m'astreins à jeter sur la page, de manière délibérément aléatoire, les mots-clés de l'épisode qui pose problème - le nom des personnages impliqués, les accessoires, les éléments du décor, tout ce qui peut avoir un sens dans l'histoire.

70 Généralement, cette mise à plat des données, leur éparpillement spatial sur le papier, me révèle des questions potentielles, des interactions inédites entre des éléments qui ne s'étaient jamais rencontrés dans mon esprit auparavant (parce que leurs articulations, jusqu'alors, procédaient uniquement d'une relation linéaire) ${ }^{2}$. En matérialisant ces nouvelles relations par des flèches qui s'entrecroisent sur le papier, je parviens généralement à réactiver l'espace négatif de l'épisode : mon schéma dissout l'alignement rigide des composants des deux scènes, et me permet de retrouver ainsi la marge de manœuvre et d'invention qui me faisait défaut.

\section{Le flux de l'information : le modèle question-réponse}

71 Ce que j'appelle le flux d'information embrasse tout ce que l'on a besoin de savoir pour comprendre les situations, les actions et les comportements des personnages de l'histoire. Afin d'éviter les pavés d'explications indigestes, il faut entièrement vectoriser ces données. L'information doit devenir un flux. Le principe est simple : avant de donner une 
explication sur tel ou tel point, il faut attiser la curiosité du lecteur. En d'autres termes, il faut l'amener à se poser, plus ou moins consciemment, la question.

Le flux progresse donc comme un jeu de questions et de réponses : un mot, une situation, un développement "pose une question" à laquelle je réponds dans une scène subséquente. Cela ressemble un peu à un jeu de taquet - ou plutôt, à plusieurs jeux de taquet se déroulant en parallèle et à des rythmes différents, car la partie se déroule à toutes sortes de niveaux (les personnages s'en posent un certain nombre, mais le lecteur peut s'en poser d'autres). Le point d'interrogation peut porter sur un détail et se résoudre à la page suivante, ou lancer une question majeure dont la réponse viendra bien plus tard et fera bifurquer l'intrigue dans une tout autre direction. À toutes les pages, il y a une ou deux cases vides qui demandent ainsi à être comblées (et qui ouvrent d'autres cases dès qu'elles sont comblées).

Il est très important de fixer les « règles » de ce jeu au plus vite avec le lecteur pour créer un effet de traction basé sur sa curiosité : s'il sait d'expérience que l'un ou l'autre mystère se résoudra au verso, il tournera la page. Je m'attache donc, dès le début de l'histoire, à soulever quelques points d'interrogation (généralement mineurs), auxquels j'apporte sans tarder des résolutions.

À première vue, le modèle question-réponse peut paraître mécanique et stérile. De fait, il risque fort de le devenir si toutes les questions sont du même ordre et s'expriment sur le même registre (comme cela arrive souvent dans les œuvres de genre, comme le film d'horreur ou le roman policier classique); le jeu devient alors prévisible ; le pli est pris, c'est toujours la même partie qui recommence.

Pour ma part, je m'efforce d'utiliser la plus large bande passante possible, en jouant avec des questions qu'un thriller, un roman d'espionnage ou un film de science-fiction ne poseraient pas et qui relèvent plutôt du récit biographique. Je déborde des conventions du genre : les questions se posent sur des plans très variés et les explications arrivent souvent de manière presque désinvolte, par un canal inattendu - et, en général, plus tôt que le lecteur ne le prévoyait. (J'essaye constamment de le prendre à contre-pied.)

Si toutes les informations sont ainsi vectorisées, aptes à stimuler la curiosité du lecteur (puis à la satisfaire), on peut aussi se permettre d'accroître sensiblement la masse d'information véhiculée par une histoire sans provoquer de fatigue. Les personnages dits «secondaires " sont les premiers à bénéficier de cette ressource «computationnelle » supplémentaire.

77 En général, la raison d'être de ce type d'acteurs est étriquée : ils n'apparaissent que pour apporter une information, pour rendre crédible une situation ou justifier un développement. (Quand on compose un scénario, on échappe difficilement à ces utilités ; pratiquement, il est impossible de s'en passer.)

78 J'essaye de les arracher à cette fonction univoque en leur attachant un petit script individuel. Chacun de ces personnages devient alors porteur d'une trajectoire propre, la plus simple possible, une histoire dont les enjeux dramatiques peuvent être exposés très rapidement et qui peut, en principe, se résoudre en deux ou trois scènes clés.

79 Bien entendu, le développement de cet arc secondaire recoupera sans cesse la ligne principale, et lui apportera un soutien précieux. Chacun de ces petits scripts individuels génère ses propres tensions dramatiques, qui donnent l'occasion de glisser certaines informations utiles et de soulever de nouveaux points d'interrogation. La trajectoire de ces personnages dans l'histoire doit être une «capsule» simple et autonome; pour 
dynamiser le récit sans le compliquer, leur script doit former un petit module à part, et parfaitement lisible.

Je construis ces personnages en piochant parmi les composants qui restent sur ma « table de travail» et auxquels je n'ai pas encore trouvé d'utilité. Des bribes d'histoires, des enjeux secondaires, des idées (de toutes sortes), que je ne parviens pas à faire entrer dans l'intrigue principale, me servent de points de départ (quitte à les laisser à l'état d'ébauche).

81 Cette gymnastique est moins arbitraire et aléatoire qu'il n'y parait, puisque tous ces éléments ont été sélectionnés d'origine pour leur potentiel dramatique intrinsèque et pour l' «air de famille " qui les rattachait à ce petit monde fictif ${ }^{3}$. Le rôle choral que jouent les personnages secondaires se rattache donc par toutes sortes de petits détails à ma table de travail initiale - à ses fantômes d'intrigues et à ses virtualités.

\section{Le rapport au dessin}

À ces fonctions typiquement "séquentielles ", il faut bien sûr ajouter toutes les autres fonctions liées à la nature visuelle et spatiale du médium ainsi qu'à la poétique spécifique du dessin, qui peut radicalement différer d'une collaboration à l'autre.

En général, si j'envisage de collaborer avec un dessinateur, c'est parce qu'une image, dans ses carnets, ou dans une histoire antérieure, est entrée en résonance avec l'un de mes "dossiers » en friche (ou a éveillé quelque envie latente). Dès que le déclic s'opère, je travaille «à l'intérieur» de son dessin, en restant en contact permanent avec les sensations qu'il me procure.

Pour pousser l'introspection encore plus loin, je dirais que je travaille dans une sorte de «langue mentale » dont le soubassement est le plaisir diffus que me procure le style du dessinateur, et ce qu'il déclenche d'associations avec mon propre imaginaire (une période, un genre narratif, une atmosphère, une problématique romanesque). Son dessin est un " prisme ». Avant même d'avoir tracé une case de l'album, le dessinateur contribue déjà à schématiser ce petit monde fictif, comme un objet 3D trop lisse dont on ferait apparaître les facettes en en limitant la résolution.

À part cela, je ne cherche pas à "visualiser » les choses outre mesure sinon quand la logique de certaines séquences implique une disposition spatiale et corporelle particulière - et dans ce cas, je fais appel à mes ressources normales de projection kinesthésiques et spatiales, celles que j'utiliserais dans la vie réelle pour me représenter un souvenir spécifique ou un projet d'action. Mais je connais assez bien, d'expérience, les limites du langage de la bande dessinée, je sais que certains gestes, certaines attitudes, certaines expressions, certaines actions se liront mal dans ce langage. J'essaye bien sûr d'éviter ou de contourner tous les écueils prévisibles.

Le but du jeu - qui reste central tout au long de l'écriture - est de fournir au dessinateur quelque chose qui va réveiller son envie de dessiner : des défis pour lesquels je fournis des solutions " assez bonnes » en espérant qu'il trouve mieux, et surtout des scènes qui vont le stimuler, l'amuser et lui donner l'envie de se surpasser. L'histoire que je lui propose a aussi pour but (assez inavouable) d'exploiter son talent; ce que je connais de son dessin me donne envie de voir certaines choses, certaines images inédites. Le scénario me donne le prétexte d'obtenir cela de lui. 
87 Je peux évidemment me tromper sur les désirs de mes dessinateurs, leur proposer des choses qu'ils n'ont vraiment pas envie de dessiner - et dans ce cas-là je suis le premier à chercher une solution de rechange. Mais je travaille en général avec des collaborateurs dont je suis proche: des amis dont je partage les goûts, les enthousiasmes, les préoccupations artistiques. Je sais quelles choses les ont mis en échec et celles qui les ont fait progresser, ou qui les ont enthousiasmés.

\section{Le dialogue}

88 Je viens de décrire tout le processus qui précède l'écriture à proprement parler. Dès que mon " plan » est fixé, je peux découper l'action et écrire le dialogue. J'entre dans un tout autre espace de création.

89 En principe, les problèmes liés à la logique de l'intrigue et à sa dramatisation sont tous résolus, je connais le point d'entrée de chaque séquence et son point de sortie : je sais sur quels enjeux dramatiques concrets elle repose, je sais quelles " questions » et quelles « réponses » je dois y activer pour faire avancer l'histoire. La nature des ellipses est bien définie. Toutes les difficultés structurelles sont donc réglées.

90 À présent, je peux m'immerger complètement dans le flux dynamique de l'histoire. J'ai le schéma théorique de ma réaction en chaîne, reste à l'appliquer concrètement. Je dois habiter chaque personnage, me demander comment il vit chaque scène, comment il réagit précisément et manifeste, par ses paroles et ses actions, les émotions que je lui ai prêtées.

91 L'écriture du scénario m'occupera quelques semaines (en général pendant des vacances). C'est la partie la plus amusante et la plus absorbante du travail: chaque séquence représente une petite équation à résoudre. Je ne vois pas passer les heures et je travaille sans pression, sans inquiétude. En y mettant le temps, je suis à peu près sûr d'arriver à trouver un «optimum local » pour chaque séquence, et toutes les petites idées de détail qui naîtront en chemin sont de l'ordre du bonus.

92 C'est aussi la phase du travail dans laquelle les possibilités expressives - et les limites - du dessin sont les plus présentes à mon esprit, car, à vrai dire, je ne sais pas du tout à quoi ressemblera la page que je décris case par case au dessinateur : je suis obligé d'avancer à l'aveuglette, en lui laissant une large part de création. Je donne des indications minimales pour la mise en scène de l'action, le cadrage, les ambiances, l'expression spécifique des personnages - très souvent, je fournis aussi des éléments de référence (bouts de films, illustrations) - mais je sais que l'impact visuel de la page terminée est impossible à prévoir. Les dessins me surprendront, forcément. Cela fait partie du plaisir de ce type de collaboration créative.

93 Parce que le dessin ne vient qu'après coup, je tends à écrire des dialogues filés, assez différents, je pense, de ceux qu'écrivent les auteurs complets qui réalisent le dessin et le scénario de manière plus ou moins simultanée (au minimum, la plupart réécrivent les scènes et les dialogues tout en dessinant). Quand j'écris le dialogue d'une séquence, je me relis constamment en repartant du début (et sans tenir compte des didascalies) pour repérer toutes les aspérités, les lourdeurs d'expression, les automatismes de langage. Ces relectures incessantes contribuent sûrement à renforcer l'aspect suivi de mes dialogues : instinctivement, j'évite le morcellement des échanges, les répliques orphelines, les exclamations isolées. 
phrases que j'emprunte sont rarement les premières qui se présentent à l'esprit pour un sens donné. Heureusement, leur baroquisme (très relatif) est compensé par la brièveté de l'expression et la clarté syntactique. C'est un langage dont le caractère artificiel est à peine perceptible car la facilité de lecture du texte garantit la fluidité d'intégration des données visuelles et verbales. Et si certaines tournures sont inhabituelles, cette légère 
déviation à la norme (le mot suivant n'est pas toujours celui qu'on attend) peut même susciter, à peu de frais, une lecture plus alerte - donc plus immersive - de la part du lecteur.

À tout cela vient s'ajouter la question (pour moi assez énigmatique) du rythme de la phrase. J'avoue n'avoir toujours pas compris exactement ce qui me pousse à tourner telle ou telle réplique dans tous les sens pour supprimer une syllabe ou une cheville qui me paraît boiteuse. J'ai beau en compter les "pieds ", je n'ai jamais percé la nature de la formule rythmique qui, dans certains cas, me fait obstinément chercher une solution différente. Je suppose que ma pratique de l'improvisation jazz joue un certain rôle en l'affaire : c'est un jeu de syncopes et d'accents, de temps forts et de temps faibles. Il n'est pas sûr qu'un acteur y trouverait son compte, ni qu'un roman puisse adopter ce genre de pattern rythmique à l'usage exclusif des dialogues. En fait je n'en sais rien : tout ce que je sais, c'est que j'écris spécifiquement pour des bulles de bandes dessinées.

The truth of the pudding is in the eating, aiment à dire les Anglais. Ce texte, conçu tout spécialement pour la revue Genesis, touche à un tabou prudemment respecté par la plupart des auteurs, qui savent qu'il est vain d'exposer publiquement ses propres méthodes de création, et impossible de le faire sans verser dans la promotion de ses recettes favorites et l'autosatisfaction. Et tout cela sous peine de rendre bien plus sévère encore le seul test critique qui compte - la lecture de l'œuvre elle-même. Pour tout avouer, mes signaux d'alerte n'ont cessé de virer au rouge en écrivant ce texte auquel je donnerais volontiers le label «à ne pas mettre entre toutes les mains». Je me suis pourtant prêté à cet exercice périlleux - et (com)plaisant - en songeant aux immenses efforts que certains chercheurs consacrent à reconstituer la méthode personnelle des auteurs par un travail d'archéologie génétique exigeant et minutieux. C'est à eux - et à personne d'autre- que j'ai voulu m'adresser ici, en leur ouvrant aussi largement que possible la porte de mon " garage hermétique ».

Ach 

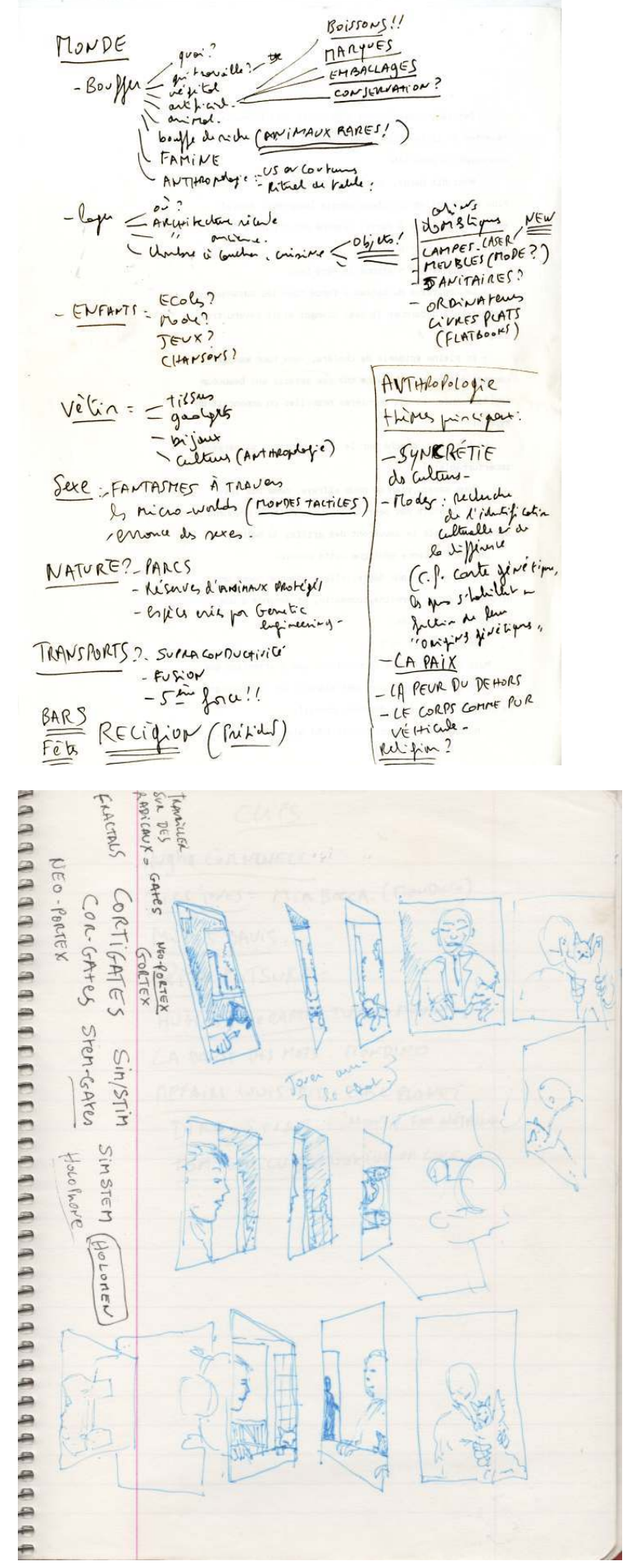


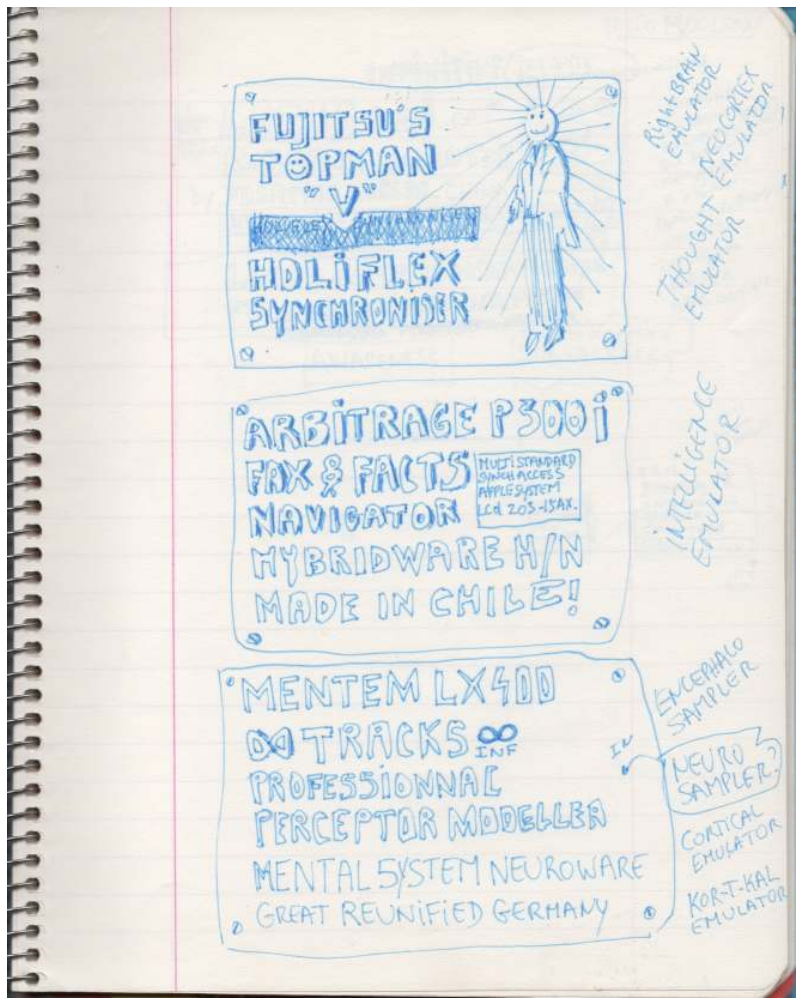



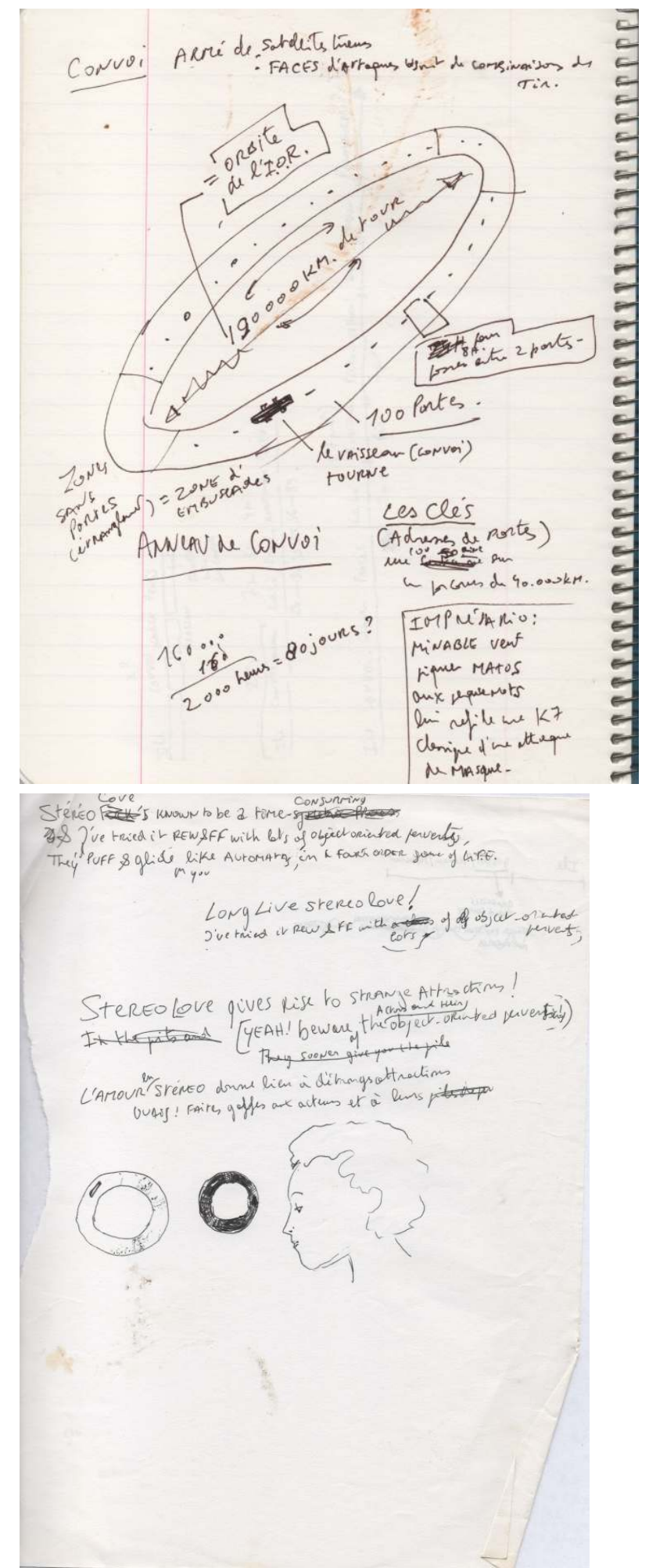

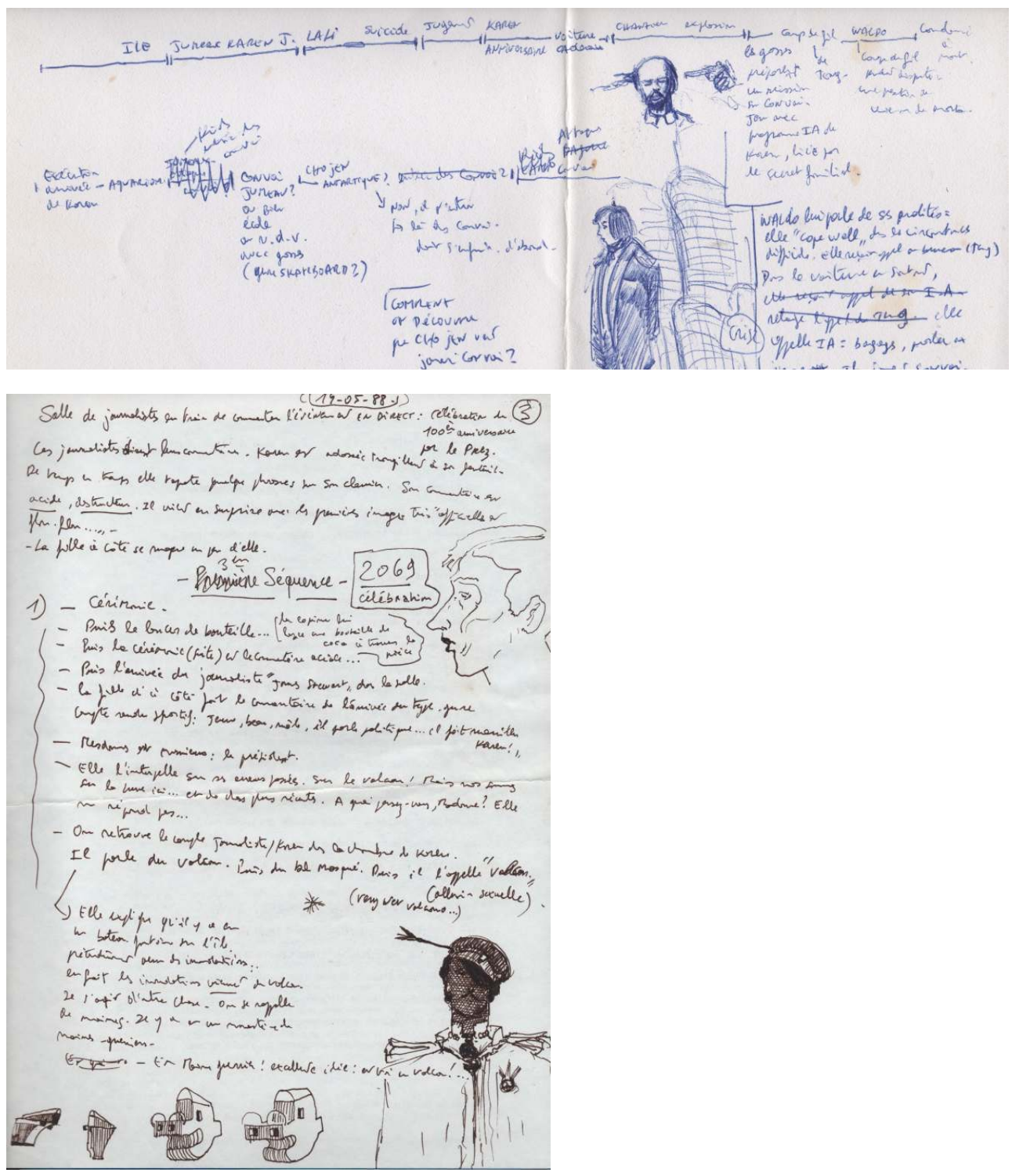

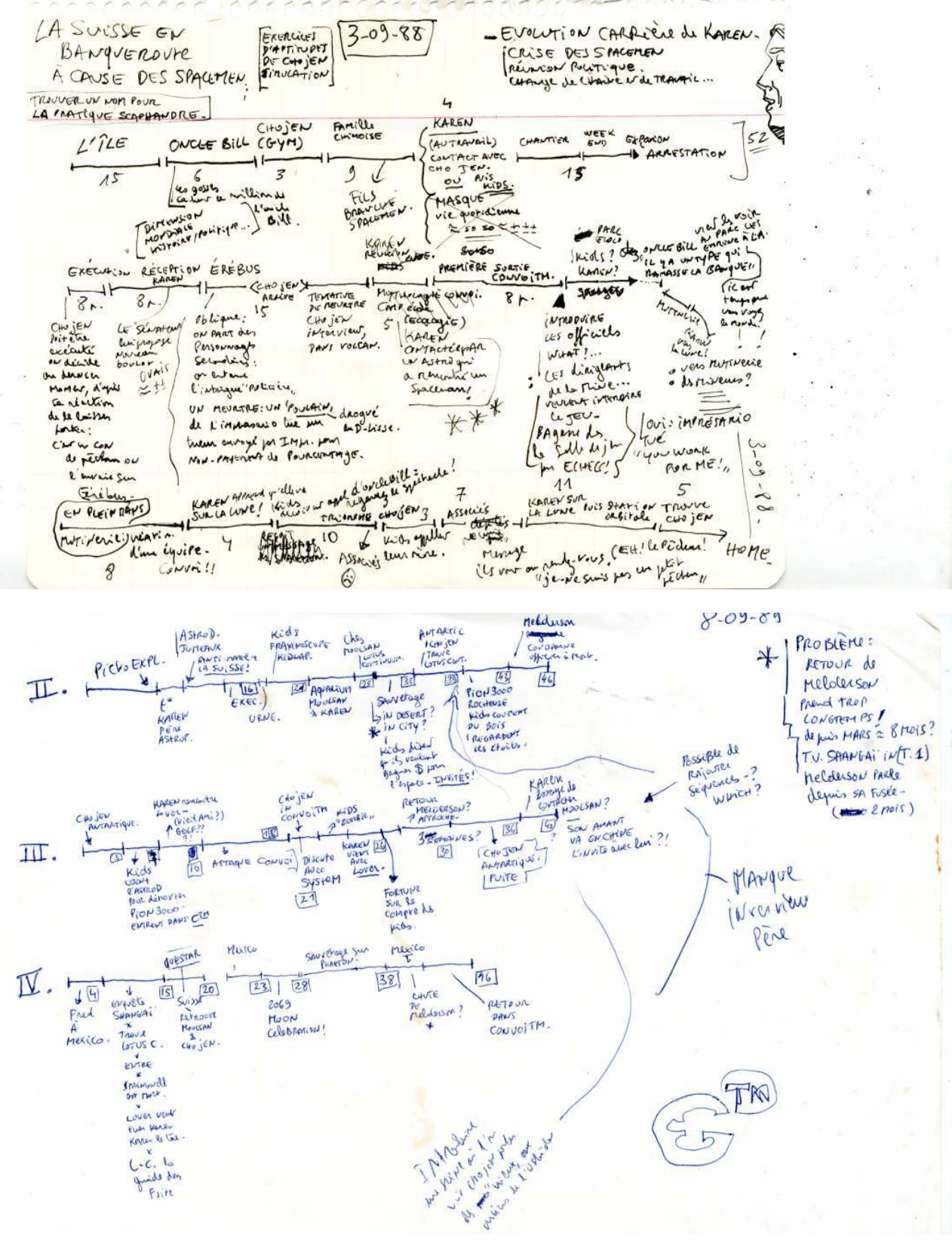

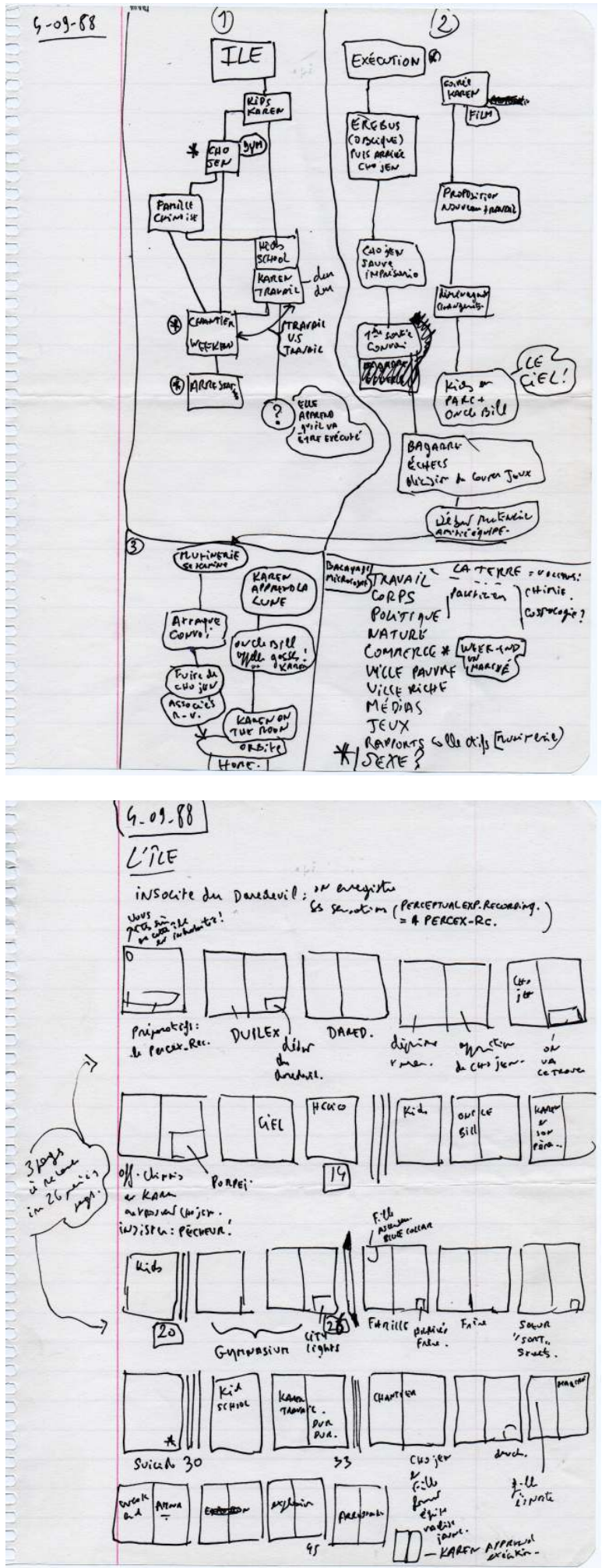


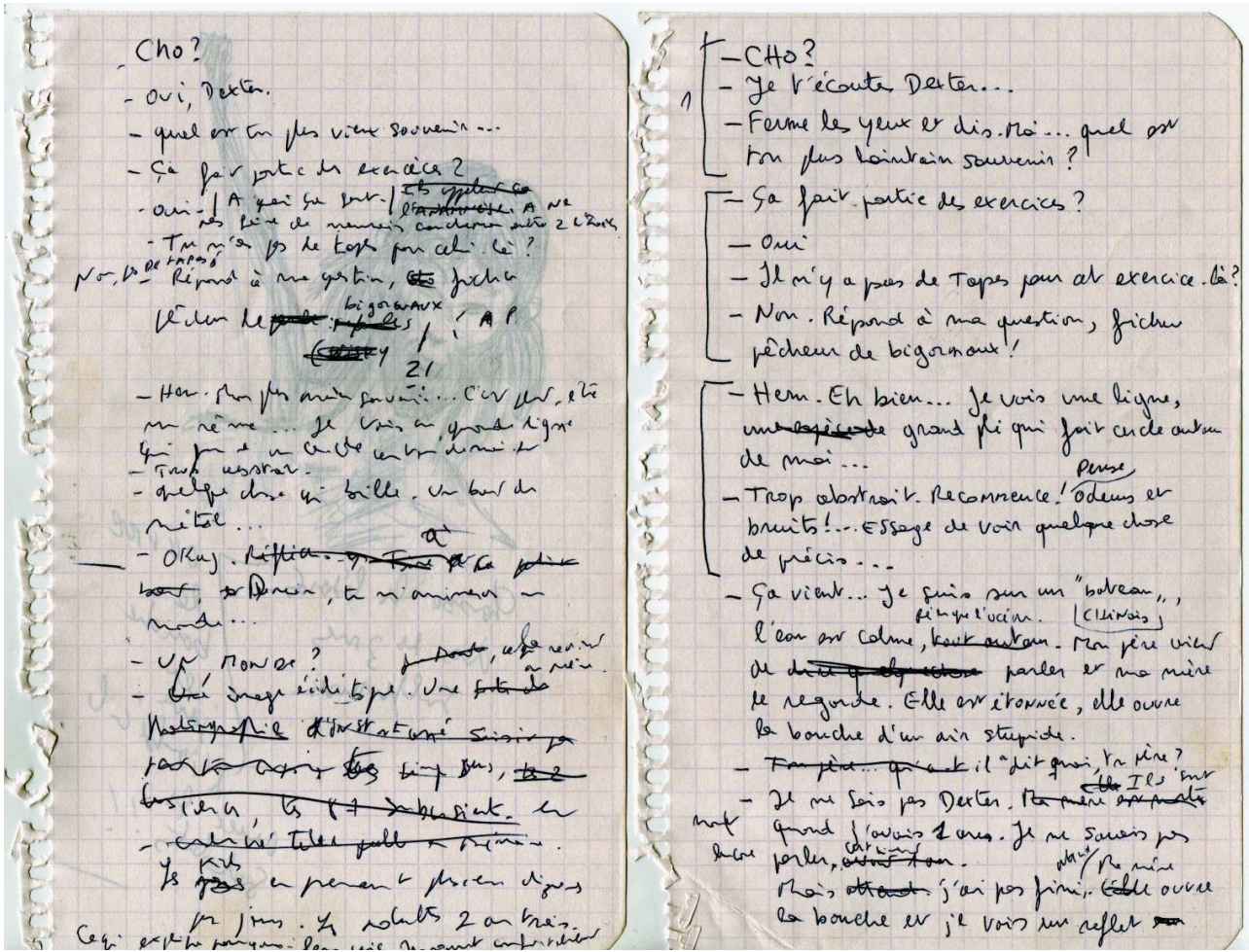

\section{ANNEXES}

Extrait du dossier génétique de la série Convoi (Thierry Smolderen, Philippe Glaucker)

Le dossier génétique de la série Convoi (sous le titre-bannière Les Aventures de Karen

Springwell), publié en quatre volumes aux éditions Les Humanoïdes de 1990 à 1995, nous a semblé révélateur des techniques scénaristiques de l'auteur, encore présentes dans ses propos sur son dernier album L'Été Diabolik.

Convoi, dans l'esprit de ses deux auteurs, est situé dans un monde futuriste (2069) saturé de technologies de pointe. Dans le travail de Thierry Smolderen, qui nous a donné accès à ce dossier très riche, il s'agit bien, par listes, par regroupements d'idées, fiche après fiche, de fixer (ou de rebrasser) les éléments anthropologiques d'un monde dont la cohérence idéologique (noms de marque, objets, armes, vêtements, transports) constitue la base d'un développement graphique à venir. L'enjeu de la cohérence visuelle des éléments et des espaces est d'autant plus cruciale que Convoi TM, qui donne son titre à la série, est un jeu vidéo en réseau où se perdent les consciences et les représentations, dans un temps où les émissions de télévision font de l'audience à partir du suicide du joueur. Les documents reproduits ci-après témoignent assez clairement de cette forge de l'idée d'image, et décrivent deux, voire trois ans avant l'édition finale, le travail acharné et 
rigoureux, méticuleux et plastique qui veut aboutir à la composition d'un monde et simultanément à la pensée critique et politique.

Fig. 1 : T. Smolderen, Convoi, dossier génétique (1988-1989); encre noire sur feuille volante A4 ; recherches thématiques et anthropologiques, caractéristiques du langage-monde à l'œuvre lors des premières étapes de réflexion

Fig. 2 : T. Smolderen, Convoi, dossier génétique (1988-1989); encre noire et bleue sur cahier rouge à spirales ; études de cadrage tridimensionnel en relation avec la notion d'holomen et d'holophone présente dans la marge gauche

Fig. 3 : T. Smolderen, Convoi, dossier génétique (1988-1989) ; études de logos et d'enseignes pour des marques destinées à figurer dans les décors urbains

Fig. 4 et 5 : T. Smolderen, Convoi, dossier génétique (1988-1989; exemple de persistance d'une idée d'univers et d'une figure (l'architecture circulaire) dans le dossier génétique, avec bifurcation vers la notion de sexualité virtuelle dans le second cas

Fig. 6 : T. Smolderen, Convoi, dossier génétique (1988-1989) ; encre bleue sur feuille volante (détail) : apparition d'une time-line sommaire, combinée avec des scènes (meurtre ou suicide) et une question

Fig. 7 : T. Smolderen, Convoi, dossier génétique (1988-1989); encre noire sur feuille volante, apparition du détail d'une séquence narrative et de fragments de dialogues, précédés de notules didascaliques et combinés à des dessins dont certains semblent relever de l'automatisme

Fig. 8 : T. Smolderen, Convoi, dossier génétique (1988-1989) ; feuille détachée d'un cahier à spirales, orientation paysage, où la time-line se complexifie et s'enrichit d'idées de scènes. En haut à droite, on notera la présence d'un profil, élément récurrent du dossier génétique

Fig. 9 : T. Smolderen, Convoi, dossier génétique (1988-1989) ; encre bleue sur feuille volante A4 orientée paysage ; mise en place des séquences globales par tome de la série (en l'occurrence II, III et IV)

Fig. 10 : T. Smolderen, Convoi, dossier génétique (1988-1989) ; encre noire sur feuille détachée d'un cahier à spirales ; reprise verticalisée et arborescente des séquences narratives apparues dans les documents précédents

Fig. 11 : T. Smolderen, Convoi, dossier génétique (1988-1989); exemple d'une conversion de la time-line et des arborescences en structure d'album par schématisation des séries de doubles pages

Fig. 12 : T. Smolderen, Convoi, dossier génétique (1988-1989); encre noire sur deux feuilles détachées du même carnet à spirales ; apparition à droite de crochets rythmiques isolant les répliques à regrouper par case, bande ou planche

\section{NOTES}

1. T. Smolderen et A. Clérisse, L'Été Diabolik, Paris, Dargaud, 2016.

2. Cet expédient m'a été directement inspiré par un documentaire sur les méthodes de Chaplin. Il vise à reproduire sur le papier quelque chose du travail d'improvisation très poussé qu'il menait avec ses comparses dans les décors du film avant même de connaitre le sujet de l'histoire.

3. Dans L'Été Diabolik, par exemple, l'intrigue secondaire du film super 8 tourné à Dallas pendant l'assassinat de Kennedy provient d'une idée de scénario très ancienne, qui, par effet de synchronie sympathique, s'est tout naturellement rattachée à l'univers sixties de Diabolik.

\section{RÉSUMÉS}

Thierry Smoderen offers a detailed synthesis of the process that for years have preceded and accompanied his making of comic strips scenarios: stages of accumulation, documentation, iconic 
research (film, photography), definition of mental territories, construction of imaginary worlds, interaction with the draftsman and anticipation of his graphic world. He also describes the elaborate stages of plot composition as a puzzle, the ramification and surprises of the narration, the energy required to avoid too obvious sequences, the spaces left open for the unexpected. Last, immersion in the flow of information generated by the sequences makes it possible to test the soundness and coherence of the whole and to ascertain the strength of the connections between the scenes on several simultaneous levels of meaning.

Thierry Smolderen propone una síntesis detallada de los procesos que preceden y acompañan en su caso y desde hace años- la fabricación del guión de una historieta : fase de acumulación, de búsquedas icónicas (cine, fotografía), definición de los territorios mentales, construcción de los universos imaginarios, interacción con el dibujante y anticipación de su universo gráfico; describe también las etapas complejas de la composición de la intriga como un rompecabezas, las bifurcaciones y las sorpresas del relato, la energía necesaria para evitar secuencias demasiado previsibles, los espacios dejados abiertos a lo inesperado. La inmersión en el flujo de informaciones generado por las secuencias permite, finalmente, poner a prueba la solidez y la coherencia del conjunto y verificar la fuerza de los vínculos entre las escenas, en varios niveles simultáneos de significación.

Thierry Smolderen propone una sintesi dettagliata dei processi che, nella sua opera, precedono e accompagnano da anni la realizzazione dei testi e delle sceneggiature di fumetti: fase d'accumulazione, di documentazione, di ricerca iconografica (cinema, fotografia), definizione dei territori mentali, costruzione dei mondi immaginari, interazione con il disegnatore e anticipazione del suo universo grafico. Sono anche descritte le tappe complesse della composizione in forma di enigma dell'intrigo, le biforcazioni e le sorprese del racconto, l'energia necessaria ad evitare le sequenze troppo prevedibili, gli spazi lasciati aperti all'inatteso. L'immersione nel flusso delle informazioni generato dalle sequenze permette infine di testare la solidità e la coerenza dell'insieme, verificando la forza dei legami tra le scene su più livelli di significato simultanei.

Thierry Smolderen legt eine detaillierte Zusammenschau jener Vorgänge vor, die im Falle seiner eigenen Produktion seit Jahren die Entwürfe zu seinen Comics begleiten bzw. den Entwürfen vorangehen: die Phase der Materialsammlung, der Dokumentation, der Bildersuche (Kino, Photographien), Abgrenzung des geistigen Territoriums, Konstruktion imaginärer Welten, Interaktionen mit dem Zeichner und Vorgriff auf dessen graphische Welt. Der Artikel berichtet auch von den komplexen Etappen der Zusammenstellung der Handlung, von Weggabelungen und Überraschungen in der Erzählung, von der Energie, die investiert werden muss, um Sequenzen mit allzu leicht vorhersehbarem Ausgang $\mathrm{zu}$ vermeiden, und von den Räumen, die für Unerwartetes offengelassen sind. Das Eintauchen in den Informationsfluss, der durch die Sequenzen entsteht, erlaubt es schließlich, die Stichhaltigkeit und die Kohärenz des Ganzen zu testen und $\mathrm{zu}$ überprüfen, ob die einzelnen Szenen auf verschiedenen simultanen Bedeutungsebenen hinreichend stark verbunden sind.

\section{AUTEUR}

\section{THIERRY SMOLDEREN}

Thierry Smolderen, né en 1954 à Bruxelles, s'est très tôt spécialisé dans l'écriture de scénarios pour la bande dessinée, avec un goût prononcé pour les séries d'albums d'anticipation : univers dystopiques de la science-fiction, aventure et invention de mondes complexes peuplés de créatures étranges et d'objets futuristes. 
Au fil des années, alors que l'auteur collabore par ailleurs aux Cahiers de la Bande dessinée, qu'il enseigne à l'EESI d'Angoulême et qu'il construit une œuvre scientifique sur la naissance de la bande dessinée (parue en 2009 aux Impressions nouvelles), la dimension politique et anticipatrice du regard de Thierry Smolderen s'accentue et apparaît de manière puissante dans la série Convoi. Aventures de Karen Springwell (Les Humanoïdes, de 1990 à 1995, avec Philippe Glaucker) puis dans Ghostmoney (série en cinq tomes, le dernier paru fin 2015, avec Dominique Bertail au dessin).

Friand de musique (toutes les musiques, mais en particulier le jazz), la composition de ces scénarios répond à la fois à une organisation stricte et à une grille d'improvisation où les horizons traditionnels d'attente sont régulièrement déjoués. Chercheur de formes et d'esthétiques nouvelles, publié chez les plus grands éditeurs français (Dargaud, Delcourt) et de nombreuses revues francophones ou anglophones, Thierry Smolderen collabore avec des dessinateurs et des coloristes aux techniques souvent très éloignées. Son scénario le plus récent, L'Été Diabolik (Dargaud), a été mis en images par Alexandre Clérisse, qui témoigne dans ce numéro de sa pratique virtuose du dessin vectoriel. Son témoignage sur les procédures d'écriture des scénarios est donc riche d'une longue expérience et particulièrement précieux pour l'étude de la génétique des scénarios en bande dessinée.

Nous lui avons demandé de revenir sur les processus d'élaboration du scénario tout au long de ces années d'écriture et de conception. 